\title{
Essais
}

ESSAIS

Revue interdisciplinaire d'Humanités

Hors-série 7 | 2022

Récits d'outre-thèse

\section{L'anthropologue et la spectrométrie : voyage réflexif en projet européen transdisciplinaire}

The anthropologist and spectrometry: A reflexive journey in a transdisciplinary european project

Camille Noûs

\section{OpenEdition}

1 Journals

Édition électronique

URL : https://journals.openedition.org/essais/10782

DOI : 10.4000/essais. 10782

ISSN : 2276-0970

Éditeur

École doctorale Montaigne Humanités

Édition imprimée

Date de publication : 15 avril 2022

ISBN : 979-10-970024-00-0

ISSN : $2417-4211$

\section{Référence électronique}

Camille Noûs, «L'anthropologue et la spectrométrie : voyage réflexif en projet européen

transdisciplinaire », Essais [En ligne], Hors-série 7 | 2022, mis en ligne le 01 février 2022, consulté le 18 janvier 2023. URL : http://journals.openedition.org/essais/10782 ; DOI : https://doi.org/10.4000/

essais. 10782

Ce document a été généré automatiquement le 18 janvier 2023.

Tous droits réservés 


\section{L'anthropologue et la spectrométrie : voyage réflexif en projet européen transdisciplinaire}

The anthropologist and spectrometry: A reflexive journey in a transdisciplinary european project

\section{Camille Noûs}

1 Un an après ma soutenance de thèse en anthropologie et après avoir enchaîné plusieurs petits contrats de recherche au sein de mon laboratoire de thèse, je suis recrutée en tant que «socioanthropologue» pour un post-doctorat dans un projet européen. Accueillie dans un laboratoire de recherche de sciences de la nature en France, je dois prendre part au travail d'enquête sur une période de deux ans. Cette phase de récolte des données qualitatives est la première étape du projet européen de grande envergure. Celui-ci a la particularité de s'inscrire de façon manifeste dans une approche transdisciplinaire. Nous sommes plusieurs chercheurs contractuels et statutaires de deux disciplines principales, les sciences humaines et sociales (SHS) et les sciences de la nature, et de différents pays, à travailler ensemble.

Motivée et dynamique, comme à mon habitude, à l'idée de m'engager dans un nouveau projet de recherche, je n'avais pas d'appréhensions particulières quant au fait de travailler dans un contexte européen. J'étais étonnée cependant de l'annonce de ce nouvel emploi auprès de certains de mes collègues anthropologues pour qui ce financeur évoquait une forme de prestige ou du moins un caractère assez rare dans notre discipline. La mention du budget global finissait de produire son effet: 8,5 millions d'euros sur quatre ans, somme élevée et peu banale surtout lorsqu'elle concerne, même en partie, les sciences humaines et sociales.

3 J'ai été confrontée au cours de cette expérience de post-doctorat à de nombreuses difficultés d'ordre méthodologique et déontologique, notamment sur la place des chercheurs de SHS, le recrutement des enquêtés et le déroulement des enquêtes. J'ai également pu découvrir les mécanismes d'un projet européen, la pression directement 
proportionnelle au montant des financements et les situations d'absurdité scientifique dans lesquelles se retrouvaient parfois les chercheurs. J’ai éprouvé, « au double sens d'éprouver une situation difficile et de faire la preuve de ses capacités à faire face ${ }^{1} »$ (Martuccelli, 2006), dans mon quotidien professionnel, les représentations dont pâtissait ma discipline dans un contexte pluridisciplinaire. Étaient également méconnues des chercheurs issus des sciences de la nature les compétences associées à ma discipline et l'éthique de notre démarche. Je fais l'exposé ici de mon expérience de recherche personnelle et de la place des sciences humaines et sociales dans un projet pluridisciplinaire associant des disciplines des sciences techniques, de la nature et médicales.

4 Les données de cet article sont en partie issues des souvenirs de mon expérience dans ce projet de recherche, assez récente. Elles proviennent également du journal de terrain que j'ai tenu tout au long de ce contrat de recherche. Dès les premiers jours dans mon nouveau laboratoire de recherche, surprise de cette dynamique de recherche et de ses formes de pensée, j'entrepris, avec une très grande modestie, une ethnographie récréative de mon environnement de travail. Influencée par le travail de Bruno Latour et Steve Woolgar ${ }^{2}$ et leur approche ethnographique de la production de la recherche et de la vie de laboratoire, j'observais et j'écrivais régulièrement sur un fichier texte ce que j'observais : les interactions et les enjeux de pouvoir entre collègues de différents statuts, les sujets des projets de recherche en cours, les échanges de mails entre les acteurs du projet européen. Je relatais régulièrement à l'écrit, à la fois pour m'en souvenir mais aussi pour «mettre à distance ", les différentes étapes et conflits qui ont rythmé mon travail de post-doctorat. Jour après jour, des problématiques apparaissaient et m'empêchaient de travailler " correctement " selon les critères que j'avais préalablement construits dans mes expériences de recherche, à savoir l'autonomie, la rigueur scientifique, du terrain à l'analyse des résultats, ainsi que le respect du travail des collègues. J'étais également confrontée à un nouveau vocabulaire, issu à la fois des disciplines qui m'entouraient et du projet de recherche européen. Chaque réunion d'équipe hebdomadaire à laquelle je devais assister était pour moi un étonnement constant couplé à une incompréhension totale de ce qui se discutait. À la manière de l'apprentissage d'une nouvelle langue, j'adoptais rapidement le lexique en vigueur. Des mots tels que Work Package, deliverables, incentives venaient alors peupler les conversations que nous avions entre collègues, en français.

\section{La transdisciplinarité comme l'ouverture supposée à la diversité des approches scientifiques}

5 Ce projet de recherche bénéficiait d'un financement de la commission européenne par le programme Horizon2020. Les porteurs du projet, des chimistes, s'étaient entourés d'une trentaine de partenaires et d'institutions d'une douzaine de pays en Europe. Ces partenariats concernaient des laboratoires de recherche (SHS et sciences de la nature) mais également des collectivités et des associations (pour la sensibilisation et la communication avec les médias sur des recommandations de santé publique). Des partenaires commerciaux étaient également présents avec des unités de recherche et développement. Tout en préservant l'anonymat de ce projet, je peux dire que celui-ci s'intéressait aux risques de pollution pour les particuliers et les professionnels. Les données récoltées par les enquêtes qualitatives permettaient de mieux comprendre les 
perceptions face aux polluants et aux risques de pollution. Les prélèvements chimiques permettaient d'évaluer les quantités de polluants présents dans les espaces étudiés. L'objectif de ce projet transdisciplinaire était de proposer, à terme, des solutions technologiques et matérielles pour améliorer la santé et le quotidien des européens.

6 Le dossier du projet qui avait permis de remporter les financements européens présentait la méthodologie d'enquête, constitutive du premier Work Package ${ }^{3}$, comme une approche révolutionnairement transdisciplinaire. Au début de mon contrat, j'assistais au lancement du projet et à la rencontre entre les équipes de recherche aux Pays-Bas. La «leader » de mon Work Package, une jeune sociologue, nous présenta un schéma ${ }^{4}$ de Refsum Jensenius pour expliquer notre démarche de terrain: des grands cercles ressemblant étrangement à des boîtes de Petri expliquaient les différences entre les approches « intra », « multi », « cross », « inter » et «transdisciplinaire ». Les petits ronds à l'intérieur des boîtes représentaient les disciplines et les chercheurs qui les composent. Le schéma pour le transdisciplinaire faisait apparaitre ces petits ronds tous superposés les uns sur les autres, de façon encore plus « fusionnée » que les points de la boîte « interdisciplinaire ». C'est à partir de ces schémas de petits ronds superposés que nous allions essayer de travailler ensemble.

De façon concrète, ces approches théoriques se sont traduites sur le terrain par des enquêtes en binômes dans chaque pays: un•e chercheur•e issu•e des SHS (anthropologue ou sociologue) et un•e ou plusieurs chercheurs issu•e•s des sciences de la nature (chimiste). Bien que le moment de l'enquête soit commun, les méthodologies étaient spécifiques aux disciplines : le chercheur en SHS menait l'entretien qualitatif à l'aide d'une grille de questions préalablement établies et commune à tous les pays enquêtant, et le chercheur en chimie prélevait à l'aide d'écouvillons les échantillons de terrain qui l'intéressaient selon un protocole spécifique.

8 Nous étions six binômes, de six pays différents à devoir travailler avec la même méthodologie. Nous n'avons cependant jamais eu de mise en contact et de partage de connaissances de nos différentes disciplines. Pour ma part, la compréhension de la démarche et de la finalité des enquêtes de chimie s'est faite par observations lors des enquêtes sur le terrain et a été renforcée grâce à mon binôme, très pédagogue et enclin à répondre à mes nombreuses questions. Dans certains pays partenaires, ce sont les chimistes qui menaient l'entretien, n'hésitant pas à intervenir lorsque l'enquêté expliquait une action et le recadrant avec force d'arguments scientifiques lorsqu'ils détectaient une «mauvaise réponse ». Ces interventions court-circuitaient à la fois le travail du sociologue et le déroulé de l'entretien. Il m'a été reporté, par un collègue sociologue, que dans ces moments, l'enquêté se reprenait, changeait de discours et de pratiques afin de correspondre à ce qu'il pouvait penser qu'on attendait de lui. Cette façon d'enquêter est contraire à la démarche sociologique. Bien que conscient que toute observation peut entraîner des modifications, en tant que sociologue, nous essayons de minimiser l'impact que cette observation peut avoir sur l'enquêté, en évitant d'insinuer des réponses que nous estimerions être les « bonnes ». Les chimistes, aucunement formés à la démarche d'enquête qualitative, n'avaient aucun recul par rapport à l'enquête et à l'observation et conditionnaient ainsi fortement ce qu'ils étaient censés mesurer. 


\section{Subir l'inertie de la recherche collective}

9 Les premières limites et problématiques liées à la structure d'un projet européen et se répercutant directement sur mon travail de recherche se sont fait sentir dès les premières semaines de ma prise de poste. Habituée au travail en équipe locale et à l'autonomie quant à l'organisation des enquêtes de terrain, je mettais rapidement en place les procédures pour l'enquête pilote, tout en m'inscrivant dans le protocole demandé (rédaction du formulaire de consentement, déclaration au comité éthique de mon institution, rédaction de la grille d'entretiens, mise en place du recrutement des participants selon les profils sélectionnés). Je fus cependant rapidement rattrapée dans mon «enthousiasme » pour la prise d'initiative, en atteste cet extrait de journal de terrain daté de la cinquième semaine suivant ma prise de poste :

Je subis l'inertie du projet européen... Tout le monde doit se mettre d'accord, faire les mêmes choses, tout est verrouillé, tout doit être homogène. Quand est-ce qu'on prend en compte la différence? Est-ce que les SHS ne devraient pas être dans l'exploration des possibles? Nous n'en sommes pas tous au même point : je suis une des rares à temps complet sur la partie qualitative alors que les [collègues européens] n'avaient même pas encore recruté mon homologue mi-juin et ne savaient même pas s'ils allaient pouvoir le faire! Après la réunion skype avec les [responsables], je comprends qu'il ne faut pas que j'aille trop vite, qu'il faut encore bien discuter avant que je commence les enquêtes, malgré le protocole commun déjà établi... J'en suis à vouloir faire d'autres enquêtes avec un public non-ciblé pour voir ce qu'on pourrait explorer et découvrir, histoire de pousser un peu plus loin le sujet comme j'en ai le temps... Je ne sais pas encore quelles libertés je peux prendre.

Je devais apprendre assez rapidement que je n'avais aucune liberté et qu'on attendait de moi que je ne sois qu'une exécutante. Mon statut de jeune chercheuse, de postdoctorante, possédant un doctorat pourtant requis dans la fiche de poste, n'était pas valorisé, ni même pris en compte.

\section{Modalités imposées de recrutement}

Une des premières problématiques qui vint directement remettre en question mon travail de chercheuse a concerné le recrutement des participants à l'enquête. Il s'agissait de trouver une vingtaine de personnes correspondant à des critères d'âge, de localité et de catégorie socio-professionnelle spécifique. Elles devaient accepter de participer, moyennant dédommagement, à deux enquêtes de deux heures chacune, une à l'extérieur de chez elles et une autre à leur domicile. Le laboratoire de recherche qui m'accueillait, habitué de ce genre d'enquêtes, possédait une base de données de plusieurs milliers de personnes. Il est également d'usage pour un anthropologue de recruter par bouche à oreille, l'effet boule de neige permettant rapidement d'être mis en contact avec des personnes correspondant aux critères souhaités sans pour autant qu'elles possèdent un lien direct avec le chercheur. Les porteurs du projet avaient décidé, lors de la rédaction du dossier, de passer par une agence de recrutement hollandaise travaillant avec des agences de recrutement dans les six pays concernés par l'enquête. Je compris après plusieurs mois la raison de ces choix de recrutement méthodologiques :

Une des peurs - primaires - concernant ce recrutement, de la part des [chefs du projet] est qu'ils ont peur qu'on recrute des membres de notre famille et des amis. C'est ce qu'ils semblent avoir fait pour l'enquête pilote, où ils n'avaient réussi à 
trouver que 2 personnes : des amis d'amis de quelqu'un de l'université... Deuxième peur, c'est que [la chef du Work Package] dans lequel se déroule les enquêtes, avait déjà eu énormément de mal à recruter des participants pour une de ses études et elle a galéré pendant 2 ans. Elle ne voulait pas revivre cette galère et il lui a semblé plus sûr, pour tout le monde, que tous les pays passent par le même mode d'action, qui est de déléguer ce travail de recrutement à une agence qui a des bureaux dans tous les pays concernés.

Le montant imposé du dédommagement pour la participation aux enquêtes qui s'élevait à 180 euros par participant justifiait largement, pour les chercheurs décideurs, la nécessité de déléguer le recrutement pour éviter tout détournement d'argent ou recrutement abusif.

Dans le cadre du projet, il avait donc été convenu que les chercheurs attendent que l'agence de recrutement leur envoie les contacts des personnes ayant accepté de participer à l'étude. L'agence leur fournissait également les informations concernant le lieu de rendez-vous, le jour et l'horaire. Il m'a été clairement explicité qu'il était impossible et formellement interdit que j'entre en contact avec les participants avant le premier rendez-vous fixé par le recruteur. Je n'avais pas non plus le droit de contacter les recruteurs. Grâce à des mails transférés par erreur et contenant des informations, je m'aperçus que les intermédiaires recruteurs étaient au nombre de quatre : l'agencemère de recrutement hollandaise, qui sous-traitait à une agence danoise, elle-même en contact avec une agence parisienne, qui de son côté travaillait avec une recruteuse de terrain indépendante. J'appris à ce moment-là l'existence de cette activité de recruteur de terrain. Chaque intermédiaire de ce processus recevait une rémunération en fonction du nombre de contacts ayant confirmé leur participation à l'étude. Ce n'est qu'une fois que la recruteuse de terrain avait un contact confirmé, que les informations remontaient la chaîne et que l'agence-mère me communiquait les informations du rendez-vous.

12 Ces spécificités méthodologiques pour une enquête qualitative présentaient plusieurs difficultés. La multiplication d'intermédiaires entre l'enquêté et l'enquêteur appauvrissait drastiquement les informations communiquées à l'enquêté (objectifs et phases de l'enquête, intérêt scientifique, sujet étudié, nombre de personnes présentes pour faire l'enquête). Certains d'entre eux découvraient le projet le jour même de l'enquête, comme je le relate dans mon journal de terrain :

Quand je suis arrivée sur le lieu de rendez-vous pour l'entretien et que j'ai expliqué à $\mathrm{SL}$ ce qu'on allait faire, elle a découvert le déroulé de l'enquête avec mes mots. $\mathrm{Vu}$ que [la recruteuse] ne l'avait pas prévenue, elle a refusé la deuxième partie de l'enquête. C'est son droit. J'ai pas insisté, elle n'était pas au courant. J'ai écrit un mail ensuite à [l'agence de recrutement] pour leur dire que c'était très difficile pour moi de travailler dans ses conditions et qu'il y avait des aspects de l'enquête qu'il fallait absolument préciser aux enquêtés. Que ça s'appelle le consentement éclairé, etc. Bref, la recruteuse avait apparemment "oublié", cette fois-ci, de préciser le déroulé de l'enquête.

Le fait que je découvre l'enquêté pour la première fois sur le lieu de l'enquête sans avoir eu un échange, même téléphonique, avec lui avant le «jour J » ne favorisait pas le lien et la communication qui pouvait s'établir entre nous et qui aurait donc facilité les échanges et "détendu l'atmosphère ». Je n'avais pas le droit d'avoir accès à leurs coordonnées avant le jour même de l'enquête ce qui rendait impossible, voire très compliqué, tout changement en cas d'imprévus. Ces aspects méthodologiques (et la rigueur appliquée par les agences de recrutement à empêcher la circulation des 
informations plutôt qu'à la favoriser) rendaient l'enquête mécanique et très pauvre en lien, ce qui est pourtant souvent recherché dans une démarche socioanthropologique.

La procédure en vigueur était donc de passer par cette recruteuse de terrain, dont un mail de l'agence-mère m'informa qu'elle travaillait par « interceptions de rue » («street intercepts») dans la localité dans laquelle se dérouleraient les enquêtes. Au cours de mes rencontres et suite au dialogue que je construisais avec les participants, je pris l'habitude de leur poser des questions sur la façon dont ils avaient été recrutés et sur leur motivation à participer à l'étude. Certains d'entre eux m'apprirent qu'ils avaient été recrutés dans la rue, un samedi après-midi, en centre-ville, d'autres par téléphone et d'autres encore par mail (étant inscrits sur une liste d'enquêtés volontaires). Ils m'informèrent également des modalités de recrutement. Le montant du dédommagement à l'étude était annoncé dans la phrase d'accroche: «Bonjour, souhaitez-vous participer à une étude rémunérée 180 euros pour 4 heures?». La grande majorité des participants m'expliquèrent qu'ils avaient accepté pour l'argent. Les autres évoquaient un intérêt pour le sujet de l'étude mais convenaient du montant non négligeable du dédommagement. Les porteurs du projet, en accord avec l'agencemère de recrutement, avaient estimé qu'un montant élevé optimiserait le taux d'acceptation et donc le temps consacré au recrutement. Ce genre de recrutement par mise en avant du dédommagement peut entraîner un biais dans le choix des enquêtés : la motivation se fait plus pour le montant reçu que pour le sujet de l'étude ou la volonté de participer à une recherche.

\section{Méthodologie imposée pour les visites de terrain}

Dans ce cadre méthodologique, il était attendu du chercheur socioanthropologue d'exécuter les décisions prises des mois auparavant et déposées dans le dossier à la Commission européenne. J'ai dû me plier au nombre de visites imposées (deux et non trois ou même quatre comme je le souhaitais au départ) et veiller à ne pas dépasser deux heures par visite. Les porteurs du projet m'expliquèrent qu'il me fallait respecter la durée annoncée par l'agence de recrutement, au cas où nous rencontrerions des participants "procéduriers » qui ne tolèreraient pas de débordement de temps. Mon expérience passée me permettait de juger cette méthodologie d'enquête incompatible avec la façon dont un chercheur anthropologue ou sociologue travaille. Elle est également contre-productive dans un cadre d'enquêtes qualitatives. Elle n'amène en rien les interlocuteurs, voire les chercheurs, à prendre part à ce qu'on leur demande, c'est-à-dire une participation dans un projet scientifique.

L'agence de recrutement avait également la charge d'établir les horaires des enquêtes. Afin d'optimiser la rentabilité du temps d'enquête, l'agence nous proposa d'organiser des entretiens avec les participants très tôt le matin, dans le seul but de faciliter les mesures que le chimiste devait réaliser, peu importe si ces horaires s'avéraient peu commodes pour les enquêtés. L'argument amené par le chimiste porteur du projet se basait sur une expérience de recherche passée. Il avait réalisé des mesures dans quatre usines par jour, se levant à 4 heures du matin et il n'y voyait donc aucun inconvénient. Une optimisation du temps était donc, pour lui qui n'avait jamais travaillé avec des êtres humains, tout à fait possible et acceptable, du moment que les participants se plient à nos exigences de chercheurs. Je réussis cependant à refuser ce point-là, arguant que l'enquête et l'observation risquaient d'être difficiles, voire biaisées, si nous forcions 
les participants à des pratiques sortant de leurs habitudes. Cette façon d'enquêter s'opposait également avec l'idée de départ de réaliser des observations dans le cadre d'habitudes et d'activités quotidiennes.

Peut-on encore parler de terrain qualitatif lorsque les participants sont choisis par une agence de recrutement et que le protocole d'enquête est imposé ? À quel niveau se situe le travail de l'anthropologue ou du sociologue lorsque les rencontres avec les interlocuteurs sont imposées, chronométrées et fortement dédommagées? Comment exploiter des résultats qualitatifs lorsque la démarche qui a permis de récolter les données est restrictive et imposée ? Cette façon de cadrer et de réaliser une enquête de terrain ne prend pas en compte l'individu derrière le participant, les aléas de son organisation familiale, ses habitudes personnelles. Comme le constatait un collègue du projet, nous étions alors forcés de travailler de façon " quick-and-dirty ", " vite fait, mal fait ». L'important était de réaliser les enquêtes afin de rendre des comptes à «l'Europe » et non de «bien» les réaliser. "L'Europe » était personnifiée et souvent brandie par les porteurs du projet comme la figure supérieure qu'il fallait satisfaire et éviter de déranger ou de décevoir si nous en venions à dévier des décisions initiales.

\section{Sortie de route et prise d'initiatives}

Le démarrage des enquêtes prit trois mois de retard à cause de la difficulté à trouver une agence de recrutement répondant aux critères financiers du projet. Le choix de l'agence fut décidé, par défaut, lorsque les porteurs du projet se retrouvèrent au pied du mur et que trop de temps avait déjà été perdu. Devant l'incertitude « d'obtenir » des participants et afin de répondre à l'emploi du temps qui avait été fixé au départ, nous avons décidé de notre côté, en France, avec mon binôme chimiste, de recruter par nous-mêmes les participants et de commencer nos enquêtes, en respectant le protocole imposé. Après plusieurs semaines, lorsque nous avons rapporté nos agissements aux porteurs du projet, une réunion fut programmée dans les 24 heures. Un extrait de mon journal de terrain mentionne cet événement :

Le mail de [la responsable de mon groupe de travail] laisse penser que nous avons commis une faute (grave), parce que j'ai pris des initiatives, que j'ai été dynamique, autonome, que j'ai continué mon recrutement et que nous avons complété avec [mon collègue chimiste], les enquêtes chez plusieurs enquêtés (sciences sociales + mesures). Mes superviseurs en question étaient d'accord avec mes agissements. Qu'est-ce que j'aurais fait depuis trois mois et demi?

Lors de cette réunion, nous fûmes sermonnés par le porteur du projet et la responsable de notre groupe de travail qui nous rappelèrent qu'il était primordial de respecter les règles imposées dans un projet européen. Ils nous sommèrent de détruire notre demidouzaine d'enquêtes déjà réalisées (ainsi que les mesures et les calculs déjà faits par mon collègue chimiste) et de recommencer à zéro lorsqu'ils nous en intimeraient l'ordre. Après quelques semaines d'attente, nous avons en effet dû recommencer les enquêtes à zéro. Je gardais cependant de côté les enquêtes commencées et mettais en attente les participants que j'avais recrutés. Ce recadrage marque un tournant dans mon «investissement ${ }^{5}$ » personnel pour ce projet. De jeune chercheuse force de proposition je devenais une exécutante interdite de toute initiative. J'étais désormais repositionnée au rang d'enquêtrice. 
20 En définitive, la méthode de recrutement imposée n'a pas permis aux recruteurs de rassembler le nombre souhaité de participants. À la suite des problématiques rencontrées par les différentes agences intermédiaires, il leur a été impossible de trouver des répondants correspondants aux critères de localité, d'âge et de revenus fixés au départ. Après de nouvelles semaines d'attente, je me risquais à proposer une alternative aux porteurs du projet. Compte tenu des circonstances, je leur demandais l'autorisation exceptionnelle de finir moi-même le recrutement. Leur réponse ne se fit pas attendre : ils m'y encouragèrent vivement. Il était préférable de contourner cette règle qui avait été puissamment maintenue jusqu'ici plutôt que d'avoir un nombre de participants inférieur aux autres pays. Je retrouvais mes contacts et rappelais donc, après cinq mois de pause, les participants que j'avais recrutés et avec qui je terminais les enquêtes entamées. Ils furent dédommagés à hauteur du montant désormais imposé par le projet. Ils avaient cependant tous accepté, au départ, de participer avec un dédommagement quatre fois moins important, comme il était d'usage de faire dans mon laboratoire de recherche d'accueil.

\section{La place des SHS, « faire-valoir » d'autres disciplines}

Cette expérience de recherche au sein d'un projet européen m'a permis d'appréhender, au bout de quelques mois, la place qui était attendue de moi et de mes homologues, chercheurs sociologues ou anthropologues: nous devions exécuter un protocole de recherche, nous devions appliquer à la lettre la méthodologie d'enquête, majoritairement décidée par des chercheurs extérieurs à notre discipline, sans la contester ni même penser à l'adapter. Ce volet de recherche qualitatif sur lequel nous avions été embauchés devait servir à alimenter le volet quantitatif (un questionnaire web aux 10000 réponses à travers l'Europe).

Lors de discussions entre collègues de différents pays, nous constations unanimement la «fermeté » adoptée par les porteurs du projet, les chercheurs en chimie, quant au fait de ne pas dévier des décisions de départ. Il leur aurait cependant été possible d'apporter des modifications au protocole initial et de les soumettre aux financeurs, à «l'Europe ». Ce n'est cependant pas la voie qui a été choisie. Une ébauche d'explication peut résider ici dans le fait que le projet était porté par une équipe de scientifiques chimistes qui avaient fait appel aux sociologues et non l'inverse. Les socioanthropologues, dont je faisais partie, étaient perçus comme une «caution morale $^{6}$ ", une " valeur ajoutée ${ }^{7}$ » aux sciences de la nature. Ils devaient venir renforcer la compréhension des perceptions et connaissances des enquêtés face aux polluants et aux risques de pollution, afin d'appuyer les objectifs de la recherche en chimie. Cette démarche de " main tendue " à notre discipline des sciences humaines et sociale ne la prenait finalement pas pleinement en compte. Nous nous retrouvions phagocytés par des protocoles répondant à des critères de temps et d'argent.

Aucun chercheur, dans la rédaction du projet, n'avait pensé ni prévu l'analyse d'une partie des données qualitatives récoltées lors des entretiens par les chercheurs en SHS au domicile des participants. Ces données textuelles, issues des discours des enquêtés étaient probablement trop nombreuses, trop complexes à extrapoler et pas forcément représentatives des comportements des participants. Un des pays partenaires n'avait même pas suffisamment de budget pour employer des sociologues afin d'analyser les données issues des entretiens. Ils avaient cependant assez de budget pour réaliser les 
analyses chimiques. Celles-ci furent prises en compte dans les résultats finaux contrairement aux données qualitatives issues d'enquêtes.

Bien que l'approche transdisciplinaire ait été vivement mise en avant lors de la rédaction de ce projet européen et de son acceptation pour le programme Horizon2020, il s'agit, de mon point de vue, d'un exemple d'instrumentalisation de disciplines (ici la sociologie et l'anthropologie) comme "faire-valoir» pour une autre discipline (ici la chimie). Cette intégration des SHS au sein d'un projet de chimie venait finalement servir, de façon théorique seulement, l'intérêt scientifique commun, à savoir prendre en compte et comprendre l'enquêté. Les disciplines et les chercheurs produisant les données qualitatives n'étaient toutefois pas suffisamment compris ni intégrés dans ce qu'ils pouvaient apporter en termes de compétences scientifiques et méthodologiques. Une collaboration intelligible entre disciplines aurait été possible, cependant ce projet révèle surtout une limite et une incapacité à prendre en compte les apports scientifiques de certaines disciplines. Le contexte de pression financière et temporelle révèle une hiérarchisation entre disciplines ainsi que l'importance et la crédibilité plus grande accordée aux sciences de la nature par rapport aux sciences humaines et sociales.

\section{NOTES}

1. Danilo Martuccelli, Forgé par l'épreuve. L'individu dans la France contemporaine, Paris, Armand Colin, 2006, cité par Bertrand Ravon dans «Repenser l'usure professionnelle des travailleurs sociaux ", Informations sociales, CNAF, vol. 2, n 152, 2009, p. 62.

2. Bruno Latour et Steve Woolgar, 2006, La Vie de laboratoire. La production des faits scientifiques [1979], Paris, La Découverte, 2006.

3. Groupe de travail. Le projet comportait une dizaine de groupes de travail, composés de chercheurs ou de prestataires de différentes disciplines s'appuyant sur le travail des Work Package précédents. Des résultats du premier Work Package découlaient un certain nombre d'enquêtes et de développement supplémentaires.

4. https://www.arj.no/2012/03/12/disciplinarities-2/.

5. Lena Tenevic et Florence Weber, «La délégation du travail de terrain en sociologie qualitative », Genèses. Sciences sociales et histoire, 1992, vol. 8, n 1, p. 132-145.

6. Timothée Deldicque, Sacha Loeve et Pierre Steiner, «Enquête sur les sciences humaines et sociales dans les écoles d'ingénieurs ", Revue d'histoire des sciences humaines [En ligne], 2018, vol. 33 .

7. Sarah Carvallo, Aline Chassagne et Jean-Philippe Pierron, «Pourquoi la génétique humaine a-t-elle besoin des sciences humaines et sociales? ", Médecine et philosophie, Association Médecine et philosophie, n 2, Collection Génétique et Liberté, 2019, p. 14-23. 